\title{
El tratamiento con bloqueantes cálcicos y a-bloqueantes facilita el pasaje de los cálculos renales
}

\author{
Calcium-chanel-blockers and a-blockers therapy facilitate urinary stone passage
}

Hollingsworth J y col. Lancet 2006; 368:1171-79.

\section{Objetivo}

Valorar la efectividad de los antagonistas cálcicos y los a-bloqueantes en el tratamiento de la nefrolitiasis no complicada.

\section{Fuente de datos}

MEDLINE, Pre-MEDLINE, CINAHL, EMBASE, revisión de citas bibliográficas, resúmenes de congresos, correspondencia a laboratorios patrocinantes y autores principales.

\section{Selección de estudios y extracción de datos}

Se seleccionaron ensayos clínicos controlados aleatorizados (ECCA) que hubieran utilizado antagonistas cálcicos ó a-bloqueantes como terapia principal. Se excluyeron las publicaciones en las que la rama placebo hubiese recibido otros medicamentos que pudieran tener efecto sobre el músculo liso (vasodilatadores, espasmolíticos, anticolinérgicos) y se aceptó la co-intervención con corticosteroides cuando éstos se hubieran usado en ambas ramas o en la rama "tratamiento". Se comparó la proporción de pacientes que eliminaran cálculos con tratamiento médico contra aquellos que lo hicieran en forma espontánea. Se realizaron análisis secundarios entre subgrupos de medicaciones.

\section{Resultados}

Fueron incluidos nueve ECCA $(n=693)$ con pacientes ambulatorios de 34 a 46 años de edad (25 a $60 \%$ mujeres). El tamaño promedio de los cálculos fue de 3,9 a 7,8 mm, alojándose la mayoría en el tercio distal del uréter. La duración del tratamiento osciló entre una y seis semanas y el seguimiento, entre dos a seis. Considerando los resultados en forma global, el $77 \%$ de los pacientes tratado eliminó cálculos vs. el $47 \%$ de los no tratados (RR 1,65; IC95\% 1,45 a 1,88) necesitándose tratar a cuatro pacientes para que uno elimine adicionalmente su lito en forma atribuible al tratamiento. Ver tabla 1.

Tabla 1: efecto del tratamiento con alfa bloqueantes y bloqueantes cálcicos en la eliminación de cálculos de la vía urinaria.

\begin{tabular}{c|c|c} 
Estudios incluidos & RR & IC95\% \\
\hline Tamsulosina vs. control & 1,52 & 1,23 a 1,86 \\
\hline$\alpha$-bloqueantes vs. control & 1,54 & 1,29 a 1,85 \\
\hline Nifedi pina vs. control & 1,51 & 1,18 a 1,94 \\
\hline Nifedi pina más corticoides vs. control & 1,90 & 1,51 a 2,40 \\
\hline
\end{tabular}

Cuando se comparó la efectividad de los a-bloqueantes y los antagonistas cálcicos entre sí, dos estudios no mostraron diferencia, mientras que uno favoreció los a-bloqueantes. El uso de corticoides influencio los resultados pero no de manera significativa.

\section{Conclusión}

El tratamiento médico aumenta la probabilidad de eliminación de cálculos medianos en transito por el tercio distal del uréter. El tratamiento farmacológico podría modificar el algoritmo de manejo de estos pacientes.

Palabras clave: Nefrolitiasis, tratamiento médico, alfa bloqueantes, beta bloqueantes, metanálisis.

Key words: urinary stones, medical treatment, alpha blockers, beta blockers, metanalisis.

Fuente de financiamiento: National Institutes of Health Research, Blue Cross Blue Shield of Michigan Foundation, Department of Veterans Affairs Health Services Research and Development Office. No se reporta participación de la industria farmacéutica.

\section{Comentario}

Atravesadas las etapas iniciales del manejo de un cólico renal (alivio del dolor, búsqueda y localización del cálculo en la vía urinaria) y siempre que el paciente no presente criterios de gravedad (dolor refractario al tratamiento, obstrucción de la vía urinaria, infección agregada) el equipo tratante debe definir la conducta a seguir si el cálculo no se elimina en forma espontánea y permanece en la vía urinaria durante el seguimiento. Esto es así para la mayoría de los litos mayores a ocho milímetros de diámetro y en menor medida para los de cuatro a ocho milímetros o "medianos" (para estos últimos la probabilidad de eliminación espontánea ronda el 50\%). En general, cuando el paciente no elimina en forma espontánea los cálculos medianos luego de un seguimiento prudencial y casi siempre para los litos grandes, los tratamientos convencionales consisten en la ruptura o extracción de los mismos mediante técnicas de litotricia extracorpórea por ondas de choque o técnicas de endourología1.
El presente trabajo resume y analiza la experiencia de varios trabajos publicados en los que las principales drogas (y dosis) utilizadas fueron tamsulosina (0,4 mg/día) o nifedipina de acción prolongada (30 mg/día) durante diez días a cuatro semanas. Vale aclarar que estos resultados coinciden con los reportados por un metanálisis reciente respecto del tratamiento con a-bloqueantes ${ }^{2}$.

\section{Conclusión del comentador}

Siempre que esté asegurado un adecuado seguimiento clínico, parece razonable iniciar tratamiento médico en el subgrupo de pacientes con litiasis residual de mediano tamaño alojada en el tercio distal del uréter, por un periodo de hasta cuatro a seis semanas antes de proseguir con las técnicas invasivas, de mayor costo y no exentas de riesgos y complicaciones ${ }^{3}$.

Gabriel Villalón [ Medico Especialista en Medicina Familiar. Servicio de Medicina Familiar y Comunitaria. Hospital Italiano de Buenos Aires. gabriel.villalon@ hospitalitaliano.org.ar ]

Villalón G. El tratamiento con bloqueantes cálcicos y a-bloqueantes facilita el pasaje de los cálculos renales. Evid Act Pract Ambul. 11(2) 41. Mar-Abr 2008. Cometado de: Hollingsworth J, Rogers M, Kaufman S y col. Medical therapy to facilitate urinary stone passage: a meta-analysis. Lancet 2006; 368:1171-79. PMID: 17011944. Disponible bajo suscripción en: http://www.thelancet.com/journals/lancet/article/PIIS0140673606694749/fulltext

\section{Referencia}

1. PROFAM URO. Curso a Distancia de Problemas Urogenitales en el Varón. Editor: Esteban Rubinstein. Directores: Guillermo Gueglio y Esteban Rubinstein. Buenos Aires: Fundación MF "para el desarrollo de la Medicina Familiar y la Atención Primaria de la Salud", 2003. Capítulo 7. Pag-161-201.

2. Parsons J, et al. Efficacy of alpha-Blockers for the Treatment of Ureteral Stones. J Urol. 2007 Mar; 177(3):983-7.

3. Glenn P. Options in the management of renal and ureteral stones. In UpToDate 15.3. Disponible en: www.uptodate.com. 